\title{
Plasma total thyroxine and free thyroxine concentrations in congenital hypothyroidism before and during treatment
}

\author{
S L Tillotson, D B Grant, A E Ades
}

\begin{abstract}
Aims: To examine the relation between plasma total thyroxine and free thyroxine in children with congenital hypothyroidism.

Methods: Regression analysis was performed on 114 cases on the paired total thyroxine and free thyroxine measurements taken from the same blood sample. Conversion equations were derived using structural relation models.

Results: A linear relation was found between $\log$ total thyroxine and $\log$ free thyroxine values. The regression slopes for values taken before treatment and values taken while patients were receiving replacement treatment were significantly different.

Conclusions: The data suggest that there is a close association between total and free thyroxine in congenital hypothyroidism, but that the relation is changed by thyroid replacement treatment.
\end{abstract}

Several authors ${ }^{12}$ have discussed the merits of plasma assays of total thyroxine (T4) and free thyroxine (FT4) in the assessment of patients with disorders of thyroid function. Pearce and Himsworth examined the relations of $\mathrm{T} 4$ to FT4 and triiodothyronine (T3) in 122 treated hypothyroid adults. ${ }^{3}$ As far as we know no study has compared these parameters of thyroid status in infants with congenital hypothyroidism. In a national study of such children a proportion had quantitative measurements of both T4 and FT4 in the same blood samples. ${ }^{4}$ We explored the relation between T4 and FT4 at diagnosis and during treatment.

\section{Methods}

The subjects were listed on the MRC Register of Children with Congenital Hypothyroidism, which records all infants born in England, Wales, and Northern Ireland between 1982 and 1987 detected by routine neonatal screening. Those born between 1982 and 1984 were followed up beyond their fifth birthday and thus have both pre-treatment and on treatment measurements of thyroid status. Those born between 1985 and 1987 have only pre-treatment measurements. All paired plasma T4 and FT4 results from the same blood specimen were included in the study.

Thirty eight infants born 1982-84 and 19 born between 1985-87 had paired values before treatment (six infants had two or more tests), providing 68 observations in all, over an age range of 0 to 154 days. Seventy eight infants born between 1982-84 had paired values on treatment (these infants contributed between one and 14 test results with a median of two results), providing a total of 266 observations with an age range of 15-2198 days of age. Twenty one infants contributed both pre- and on treatment values.

The complete sample is therefore from 114 cases, providing 334 observations. The specimens had been analysed at several laboratories. The laboratory at Belfast was responsible for $40 \%$ of the assays and the other laboratories each contributed $13 \%$ or less.

A $\log$ transformation of T4 $(\ln$ T4) and FT4 (ln FT4) was carried out to stabilise the variance. The correlation coefficient was 0.87 after the log transformation, compared with 0.75 before.

Tests undertaken before treatment measure only naturally produced thyroxine; those undertaken while on replacement treatment measure both natural and prescribed thyroxine. It was therefore necessary to check whether the Ln T4/Ln FT4 regression slope was significantly different in the pre-treatment and on treatment groups, using a test for interaction. As both T4 and FT4 assays are subject to error, and as there was no compelling reason for choosing which should be considered the independent variable in the regression equation, conversion equations were derived using structural relation models. ${ }^{56}$ This is a generalisation of regression analysis in which it is assumed that there is error in both T4 and FT4 measurements.

\section{Results}

A scatterplot of $\ln$ T4 $v \ln$ FT4 values is shown in fig 1 which differentiates measurements obtained before treatment and on treatment. The regression slope in the pre-treatment group was significantly steeper than that in the on treatment group (interaction $\left.F_{1,333}=46.18 ; p<0.005\right)$. This remained true even after the two observations $A$ and $B$ in fig 1 , which could be considered potential outliers, were removed $\left(F_{1,331}=32.38\right.$; $\mathrm{p}<0.005)$. The difference in slopes was also still significant after values of T4 less than 20 (ln $\mathrm{T} 4<3)\left(\mathrm{F}_{1,329}=20.0 \mathrm{p}<0.005\right)$, and less than $30(\ln T 4<3.4)\left(F_{1,325}=12.13\right.$ $\mathrm{p}<0.005)$ were removed. All observations were therefore retained in the sample.

The difference in slope in the pre-treatment and on treatment groups (interaction) per- 




Figure 1 Plasma total thyroxine (T4) and free thyroxine (FT4) concentrations (log values) from 114 children with congenital hypothyroidism; pre-treatment measurements $(n=68)$ and measurements taken while on replacement treatment $(n=226)$.

sisted after accounting for the fact that a proportion of observations were from the same patient, and after controlling for the age at which the blood samples were taken, and for a possible interaction between the T4/FT4 slope and age. The difference in slope could not be accounted for by the confounding effect of laboratory. The laboratory which carried out most of the assays contributed $43 \%$ of the preand $40 \%$ of the on treatment data. Furthermore, the LnT4/LnFT4 regression slopes for

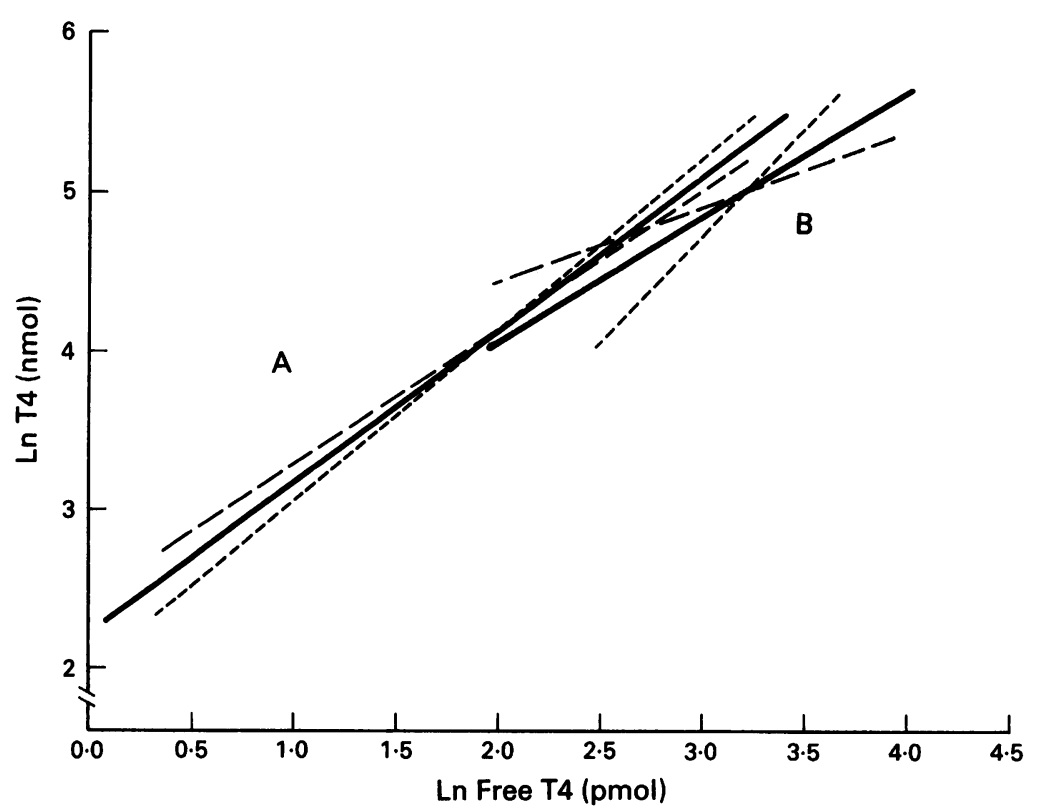

Figure 2 Conversion equations (solid lines) based on structural relation models $(A)$ Pre-treatment: In T4 $=2 \cdot 219+0.9573 \ln$ FT4 (B) On treatment: $\ln T 4=2 \cdot 522+$ 0.7729 In FT4. The more conventional regression lines based on the assumption that all the error lies in either the Ln T4 (_ - -) or the Ln FT4 (_- -) values are shown for comparison. this laboratory were not significantly different from the slopes given by the rest of the data $\left(t_{328}=0.427 ; p>0.6\right)$.

We conclude that the relation between $\mathrm{T} 4$ and FT4 may be different in hypothyroid children receiving thyroxine to that in untreated children. To assess the scale of the differences at different T4 and FT4 values structural conversion equations were calculated for the pre-treatment and on treatment groups (fig 2). An FT 4 of $10 \mathrm{pmol} / \mathrm{l}$ in untreated subjects was equivalent to a T4 value of $83.4 \mathrm{nmol} / 1$; in treated subjects this was equivalent to a $\mathrm{T} 4$ of $73.8 \mathrm{nmol} / 1$. At an FT4 of $20 \mathrm{pmol} / 1$ the difference in equivalent $\mathrm{T} 4$ was even larger $(161.9 \mathrm{nmol} / 1 \mathrm{compared}$ with $126 \cdot 1 \mathrm{nmol} / \mathrm{l})$. It should be emphasised that the conversion equations cannot be extrapolated beyond the limits of the scales.

\section{Discussion}

The results indicate that there was a close linear relation between log total thyroxine and $\log$ free thyroxine values, but that the regression slopes for the pre-treatment results and results obtained during replacement treatment were significantly different. The pre-treatment equation had a steeper slope than that for the on treatment group, suggesting that there is a higher total thyroxine to free thyroxine ratio pre-treatment. However, within the specified range the result of using our conversion equation for the on treatment group would accord closely with values derived from the equation given by Himsworth and Pearce ${ }^{3}$ in which adults on treatment with 1-thyroxine were studied, although their equation was calculated without $\log$ transformation of the data.

There are several possible confounding factors in the above study which we were not able to take into account. For example, the ratio between total and free thyroxine will vary in different subjects as a result of differences in the concentrations of thyroxine binding proteins and variations in the radioimmunoassay methods used in different laboratories. Confirmation of our conclusion that treatment alters the relation between total and free thyroxine would require a prospective study.

We thank the paediatricians and laboratory staff for providing data to the Register, the staff of the Hypothyroid Register for collecting and collating the data, and Dr Isabel Smith for her help and advice. The work was supported by a special project grant from the Medical Research Council.

Pearce CJ, Byfield PGH. Free thyroid hormone assays and thyroid function. Ann Clin Biochem 1986;23:230-7.

2 Tuttlebee JW, Bird R. A comparison of free thyroxine concentration and free thyroxine index as diagnostic tests of thyroid function. Ann Clin Biochem 1981;18:88-92.

3 Pearce CI, Himsworth RI Total and free hormone concentrations in patients receiving thyroxine. Br Med F 1984;288:693-5.

4 Grant DB, Smith I Survey of neonatal screening for primary hypothyroidism in England, Wales, and Northern primary hypothyroidism in England, Wales, and

5 Lewis PA, Jones PW, Polak JW, Tillotson HT. The problem of conversion in method comparison studies. Appl Statist $1991: 40: 105-12$.

6 Kelly GE. Use of the structural equations model in assessing the reliability of a new measurement technique. Appl Statist 1985;34:258-63. 